\title{
BMJ Open COVID-19 and perinatal intimate partner violence: a cross-sectional survey of pregnant and postpartum individuals in the early stages of the COVID-19 pandemic
}

Katherine A Muldoon (D) , ${ }^{1,2}$ Kathryn M Denize, ${ }^{1}$ Robert Talarico, ${ }^{1}$ Carlie Boisvert, ${ }^{1,3}$ Olivia Frank, ${ }^{1,4}$ Alysha L J Harvey, ${ }^{1}$ Ruth Rennicks White, ${ }^{1}$ Deshayne B Fell,,${ }^{5,6}$ Meagan Ann O'Hare-Gordon, ${ }^{1}$ Yanfang Guo (1) , ${ }^{1,7}$ Malia S Q Murphy, ${ }^{1}$ Daniel J Corsi (D , , ${ }^{1,6}$ Kari Sampsel, ${ }^{1,8}$ Shi Wu Wen (D) , ${ }^{1,5}$ Mark C Walker, ${ }^{1,2}$ Darine El-Chaar (iD) 1,3

To cite: Muldoon KA, Denize KM, Talarico R, et al. COVID-19 and perinatal intimate partner violence: a crosssectional survey of pregnant and postpartum individuals in the early stages of the COVID-19 pandemic. BMJ Open 2021;11:e049295. doi:10.1136/ bmjopen-2021-049295

- Prepublication history and supplemental material for this paper is available online. To view these files, please visit the journal online (http://dx.doi. org/10.1136/bmjopen-2021049295).

Received 21 January 2021 Revised 13 March 2021 Accepted 08 April 2021
Check for updates

(c) Author(s) (or their employer(s)) 2021. Re-use permitted under CC BY-NC. No commercial re-use. See rights and permissions. Published by BMJ.

For numbered affiliations see end of article.

Correspondence to Dr Katherine A Muldoon; kmuldoon@ohri.ca

\section{ABSTRACT}

Objective The objectives of this study were to: (1) document violent and controlling behaviours within intimate partnerships during the perinatal period; and (2) determine individual, interpersonal and household-level factors influencing the risk of perinatal intimate partner violence (IPV).

Design Cross-sectional survey.

Setting The Ottawa Hospital, Department of Obstetrics and Gynecology, Ottawa, Ontario, Canada.

Participants Patients who gave birth at The Ottawa Hospital and were $>20$ days post partum between 17 March and 16 June 2020.

Main outcomes and measures Perinatal IPV was defined as regular controlling behaviours or act-based forms of emotional/physical/sexual abuse in the 12 months before pregnancy, during pregnancy and/or post partum. Log-binomial multivariable regression models were used to compute adjusted risk ratios (aRRs) and 95\% Cls to identify potential risk factors for IPV: maternal age, postpartum depression, parity, increase in partner substance use and household income.

Results Among 216 participants, the median maternal age was 33 years (IQR: 30-36). In total, 52 (24.07\%) reported some form of perinatal IPV, $37(17.13 \%)$ reported regular controlling behaviour and $9(4.17 \%)$ reported both. Household income below the municipal median was the strongest risk factor for perinatal IPV (aRR: 3.24, $95 \% \mathrm{Cl}: 1.87$ to 5.59). There was no apparent association between maternal age (aRR: $0.99,95 \% \mathrm{Cl}: 0.94$ to 1.04), postpartum depression (aRR: $1.03,95 \% \mathrm{Cl}: 1.00$ to 1.07 ), nulliparity (aRR: $1.18,95 \% \mathrm{Cl}: 0.71$ to 1.97 ) or increases in partner substance use (aRR: $0.73,95 \% \mathrm{Cl}: 0.42$ to 1.25 ) with IPV.

Conclusion One in four individuals in this study experienced perinatal IPV. Household income was the strongest risk factor, and surprisingly, many hypothesised risk factors (eg, mental health, partner substance use, etc) were not significantly associated with perinatal IPV in this sample. This highlights the challenges in both measuring
Strengths and limitations of this study

- This study uses a detailed definition of perinatal intimate partner violence (IPV) including regular controlling behaviours, emotional, physical and sexual abuse during three perinatal time periods: prepregnancy, prenatal and postpartum periods.

- There is detailed information on household stressors and changes directly attributable to the COVID-19 pandemic protocols.

- There is detailed information on ethnicity, socioeconomic status and partner characteristics.

- Self-selection and non-response bias is a source of selection bias and under-reporting of IPV and residual confounding is a source of measurement bias.

- This study does not have a comparison group of participants prior to COVID-19 and is unable to estimate changes in perinatal IPV directly attributable to the stress of the COVID-19 pandemic.

IPV and identifying individuals exposed to perinatal IPV during the high stress of the COVID-19 pandemic.

\section{INTRODUCTION}

The evolving COVID-19 pandemic continues to cause extreme stress, unease and fear, all factors that can increase the risk of intimate partner violence (IPV) ${ }^{1-5}$ IPV broadly encompasses any form of emotional abuse, threatening behaviour, psychological harm, physical violence or sexual violence from a current or former intimate partner or spouse. ${ }^{6}$ Shortly after the initial declaration of COVID-19 as a global pandemic on 11 March 2020, the United Nations released a statement on 27 March 2020 warning of increased risks of IPV, ${ }^{7}$ in addition to the health-related 
consequences of COVID-19 infection. ${ }^{8-10}$ It is estimated that over $30 \%$ of women have experienced IPV in their lives ${ }^{11}$ and $3 \%-9 \%$ of individuals experience perinatal IPV, defined as violence or abuse that occurs 12 months prior to pregnancy, during pregnancy and up to 1 year post partum. ${ }^{12}$ To date, there are limited data on the prevalence and risk factors of perinatal IPV during the COVID-19 pandemic, despite the rising global concern for both pregnant people and the increase in violence.

The primary sources of data on violence during the COVID-19 pandemic have come from emergency departments (EDs), policing or crime data, and online surveys. Most EDs saw drastic decreases in all-cause admissions immediately following the COVID-19 lockdown. ${ }^{13-15}$ Investigations of cause-specific ED admissions varied, where some studies identified increases in IPV or assaultbased cases, ${ }^{16} 17$ while others reported decreases. ${ }^{18} 19$ Mixed patterns have also been observed in crime data from police departments, where some settings reported increases in domestic violence cases, ${ }^{20-22}$ some found decreases ${ }^{23} 24$ and others identified no detectable changes. ${ }^{2526}$ An online survey of over 2000 cis-gendered women from the USA found that $16 \%$ had experienced IPV since the beginning of 2020 , including $11 \%$ being emotionally abused, $5 \%$ forced to engage in unwanted sexual activity and $6 \%$ physically harmed. ${ }^{27}$ Another survey of over 2400 Americans found that $18 \%$ had a history of IPV, and of those, $17 \%$ reported that IPV had gotten worse since COVID-19 began. ${ }^{28} \mathrm{~A}$ representative sample of over 2000 adults in New Zealand found $10 \%$ experienced some form of sexual, physical or emotional abuse during the first month of the COVID-19 pandemic. ${ }^{29}$ Collectively, these studies highlight the complexities of assessing and evaluating patterns of violence and IPV during the pandemic, however none have included information on pregnancy or postpartum status, limiting our understanding of perinatal IPV during COVID-19.

Adverse maternal and infant outcomes associated with COVID-19 infection include higher risk of preterm birth, caesarean birth, and some cases of fetal and maternal death. ${ }^{30-36}$ A recent US Centers for Disease Control surveillance report of 24434 pregnant individuals with COVID-19 infection identified higher risk of admission to intensive care, invasive ventilation and death compared with non-pregnant individuals of reproductive age. ${ }^{37}$ A systematic review of maternal mental health during COVID-19 found significant increases in risk of anxiety and higher scores on the Edinburgh Postnatal Depression Survey (EPDS) among pregnant and postpartum individuals during COVID-19 compared with pre-COVID-19. ${ }^{38} \mathrm{~A}$ new scale, called the Pandemic Related Pregnancy Stress Scale, was developed and found that a history of abuse was an independent predictor of moderate or severe anxiety during COVID-19. ${ }^{39}$ To our knowledge, this is the only study that captured information on historical abuse among a perinatal sample; however, there was no information on perinatal IPV.
Given the limited evidence base on perinatal IPV during COVID-19 and growing concern about maternal health and safety, the goal of this study was to investigate perinatal IPV among those who gave birth during the COVID-19 pandemic. The objectives of this study were to: (1) document violent and controlling behaviours within intimate partnerships during the perinatal period; and (2) determine individual, interpersonal and householdlevel factors influencing the risk of perinatal IPV.

\section{METHODS}

\section{Study setting and context}

This study took place in Ottawa, Ontario, the fourth largest city in Canada with a census metropolitan population of 1.3 million. The provincial government declared a state of emergency on 17 March 2020. As a result, most public establishments were closed (eg, schools, childcare centres, libraries, recreational centres, restaurants, etc) and most workplaces transitioned to remote work, where possible. At the time of the study (June 2020), the cumulative number of confirmed COVID-19 cases in Ottawa was 2650 (40 161 cases in the province of Ontario, and 119451 cases in Canada). ${ }^{40}$

This study was conducted at The Ottawa Hospital, a multisite tertiary-care facility with two obstetrical wards across the city. The Department of Obstetrics, Gynaecology and Newborn Care implemented safety protocols whereby all pregnant patients underwent symptomatic screening for COVID-19 at the hospital entrance and again upon entry to the Maternal and Newborn Care floor. Care providers wore full personal protective equipment at all times (eg, universal masking) as part of the hospital-wide policy. A partner or support person could only enter once (ie, no in and out privileges) after screening negative for COVID-19. Partners were not able to attend caesarean births in the operating room. After birth, patients were not allowed to leave their hospital room for any reason and no additional children or family members were allowed to visit. For infants admitted to the neonatal intensive care unit (NICU), only one parent could be present at a time.

\section{Study design and recruitment}

This is a cross-sectional survey of patients who gave birth at The Ottawa Hospital. Patients were identified through the hospital birth records and contacted for a one-time survey if they met the following inclusion criteria: had given birth after 17 March 2020, >20 days post partum, 16 years of age or older and consented to the hospital's Permission to Contact Program. Patients were excluded if their pregnancy resulted in a stillbirth or neonatal death and were not contacted. We chose 20 days post partum as the cut-off to allow for at least 20 days to pass where postpartum IPV could occur. To improve response rate, eligible patients were contacted by phone and after obtaining verbal informed consent, a link to the online survey was sent to a private email address. This allowed for 
private completion of the survey on a personal computer or device. The survey took $10 \mathrm{~min}$ to complete. All participants were provided with links to community resources for IPV, maternal support or encouraged to contact the hospital for referrals.

\section{Patient and public involvement}

The survey and all study materials were developed in collaboration with a patient partner (MAO'H-G), who was admitted to hospital during the COVID-19 pandemic and unable to see her partner and children for 5 weeks (22 April-27 May 2020). She gave birth to preterm twins who were admitted to the NICU for 19 days. ${ }^{41}$ The patient partner was not involved in recruitment. Participants will not be directly contacted with the results of the study because of the sensitive nature of the project; however, public dissemination of the findings is available through the institutional online platforms.

\section{Outcomes}

Perinatal IPV was measured by two scales from the WHO multicountry study on domestic violence. ${ }^{42}$ Scale one, comprised of eight questions, measures different forms of regular controlling behaviour exhibited by the partner (eg, insist on knowing where you are at all times, try to keep you from seeing your friends, are often suspicious that you are unfaithful, etc). Scale two measures act-based forms of IPV including emotional abuse (ie, insulting, belittling, intimidating, threatening to hurt someone you care about), physical abuse (ie, slapped, pushed, hit, strangled, threatened with a weapon), and sexual abuse (ie, forced to have unwanted sexual intercourse, forced to have other unwanted sexual activities, forced to engage in unwanted sexual activities they considered degrading or humiliating). The act-based forms of perinatal IPV were asked for each perinatal time period: 12 months before pregnancy, during pregnancy and post partum. A composite outcome of any perinatal IPV was defined as experiencing any regular controlling behaviour (scale one) or any act-based forms of IPV (scale two) during the perinatal period.

\section{Covariables}

Demographic characteristics included age of mother (in years) and age of infant (in days) at the time of the survey. The following maternal ethnicities are presented: white people, Asian (including South, Southeast, East), Middle Eastern, black people and another person of colour. ${ }^{43}$ Participants were asked if they were born in Canada or had immigrated from another country. Participants identified any languages they feel comfortable speaking (ie, English, French or another language). Marital status compared those who were married/common law versus single/another status. Education level was measured as completing a college diploma or undergraduate university degree or higher. Participants reported if they owned their dwelling (vs rented) and their combined household income (before taxes), which was dichotomised as either at or below the median total household income for the Ottawa region ( $\$ \mathrm{C} 119440$ ) as determined by the Canadian Census. ${ }^{44}$

COVID-19-related household stress was measured by loss of income due to COVID-19 protocols (ie, permanent job loss, temporarily laid off or reduced hours) or if their partners were essential workers, defined as those who were required to continue working during lockdown to maintain the city infrastructure. Participants were asked if their partner had increased substance use since COVID-19 began (ie, alcohol, cannabis, tobacco, another substance). Changes to childcare were measured as children stopped going to school or daycare, no changes to childcare, or no children.

Maternal experiences of COVID-19-related isolation included not being able to have a baby shower (or other planned celebration), a family member could not come and stay with them as planned, friends and family could not visit the new infant, or they missed out on community resources (eg, mom-baby groups, breastfeeding support groups, play dates).

Measures of maternal mental health include preexisting anxiety, pre-existing depression, defined as receiving any counselling or treatment for anxiety or depression. Postpartum depression was measured using the EPDS. The EPDS is the most reliable and widely used screening tool for postpartum depression. The 10-item scale ranges from 0 to 30 and a score of 13 or greater on the EPDS indicates a high likelihood of depression and further assessment/management is needed. The score was presented continuously and dichotomised at 13 or greater to indicate postpartum depression. If a participant scored greater than 13 or indicated risk of suicidality (item 10), the principal investigator (DE-C, obstetrician) was notified within 24 hours of survey completion for a chart review and clinical follow-up.

Participants reported on their in-hospital care experience including if they had to wear a mask during delivery and if they were alone without a support person for the whole admission (including early labour, active labour/ delivery and post partum). Participants were asked if they had any postpartum visit to an ED for mother or infant.

\section{Analyses}

All analyses were conducted using SAS V.9.4. ${ }^{45}$ In accordance with privacy guidelines, all cell sizes $<5$ were suppressed to ensure non-identification. The characteristics of the sample were summarised using descriptive statistics including frequencies and percentages for categorical variables. Continuous variables were summarised using median and IQR, which are more robust measures and less sensitive to outliers. The characteristics of the sample were compared between those who had experienced perinatal IPV versus those who had not by calculating $\mathrm{X}^{2}$ tests for categorical variables and Wilcoxon rank-sum test for continuous variables.

Log-binomial regression models were calculated to investigate the association between five prespecified risk 
factors and perinatal IPV using unadjusted risk ratios (RRs) and 95\% CIs. The risk factors were selected to capture individual, interpersonal and household-level factors that influence risk of violence including: maternal age, EPDS, parity, increases in partners' substance use and household income below the municipal median. All covariables of interest were included in the multivariable model and presented using adjusted RR (aRR) and 95\% CI.

Seven participants had at least one missing EPDS component. To compute the EPDS score, we imputed missing components with the mean of the participant's non-missing components. In the multivariable model, missing data for household income were imputed by multiple imputation using chained equations and models were averaged across 10 imputed datasets. ${ }^{46}$

To evaluate the robustness of the analyses, we conducted a sensitivity analysis to remove participants from the sample who reported pre-pregnancy act-based forms of IPV alone (ie, no controlling behaviours, no pregnancy or postpartum act-based forms of IPV) to assess concerns of perinatal IPV that occurred before COVID-19 began.

\section{RESULTS}

Between 17 March and 16 June, 1568 individuals gave birth at The Ottawa Hospital. Of those, 613 agreed to be contacted for research, 572 had valid phone numbers and were contacted, 302 consented to the study and 261 started the survey, for a response rate of $42.58 \%$. After excluding those who did not finish the survey, the final analytical sample was 216 participants. No participants in the study reported current or previous COVID-19 infection.

There were $52(24.07 \%)$ participants who reported perinatal IPV. In total, $37(17.13 \%)$ reported regular controlling behaviours from their partners, 24 (11.11\%) reported act-based forms of IPV and $9(4.17 \%)$ reported both (table 1). The most common forms of regular controlling behaviours from partners were that they 'insist on knowing where you are at all times' (8.33\%), 'try to keep you from seeing your friends' $(5.58 \%)$ and 'get angry if you speak with another man or person they would feel jealous of' (4.65\%) (figure 1, online supplemental appendix 1 for data tables).

Figure 2 displays the frequency of act-based forms of perinatal IPV experienced by participants during each window of the perinatal period. In total, $13(6.05 \%)$ experienced any form of IPV during the 12 months prior to pregnancy, 11 (5.12\%) during pregnancy and 15 $(6.98 \%)$ during post partum. The most common form of IPV reported by participants across all time periods was emotional abuse $(10.23 \%)$. Sexual abuse was reported by $2.33 \%$ of participants, and $<5$ reported physical abuse (see online supplemental appendix 2 for data tables).

The demographic characteristics of the sample are presented in table 2. The maternal median age was 33 years (IQR: 30-36) and the infant median age at the
Table 1 Different forms of perinatal violence and combinations

\begin{tabular}{|c|c|}
\hline Perinatal IPV measures & $\begin{array}{l}\mathrm{N}=216 \\
\mathrm{n}(\%)\end{array}$ \\
\hline \multicolumn{2}{|c|}{$\begin{array}{l}\text { Act-based forms of perinatal IPV or regular controlling } \\
\text { behaviour }\end{array}$} \\
\hline Yes & $52(24.07)$ \\
\hline No & $164(75.93)$ \\
\hline \multicolumn{2}{|c|}{ Act-based forms of perinatal IPV } \\
\hline Yes & $24(11.11)$ \\
\hline No & $192(88.89)$ \\
\hline \multicolumn{2}{|c|}{ Regular controlling behaviours } \\
\hline Yes & $37(17.13)$ \\
\hline No & $179(82.87)$ \\
\hline \multicolumn{2}{|c|}{ Acts of perinatal IPV and regular controlling behaviour } \\
\hline Yes & $9(4.17)$ \\
\hline No & $207(95.83)$ \\
\hline \multicolumn{2}{|c|}{ Frequency of act-based forms of perinatal IPV } \\
\hline Single time period & $13(6.02)$ \\
\hline Multiple time periods & $11(5.09)$ \\
\hline No acts of perinatal IPV & $192(88.89)$ \\
\hline
\end{tabular}

IPV, intimate partner violence.

time of the survey was 76 days (IQR: 66-90). The sample was diverse with $147(68.06 \%)$ identifying as white, 31 (14.35\%) South/Southeast or East Asian, 18 (8.33\%) Middle Eastern, 17 (7.87\%) black, and 25 (11.57\%) identifying with another race or ethnic group. There were 151 (69.91\%) participants who owned their homes and 80 (37.04\%) had household incomes below the municipal median.

Owing to COVID-19 restrictions, 64 (29.63\%) reported household income loss and $55(25.46 \%)$ reported their partner was an essential worker who continued to work on the front line. There were $45(20.83 \%)$ participants who reported that their partner had increased substance use since COVID-19 began. Regarding maternal mental health, $43(19.91 \%)$ had pre-existing anxiety and 29 $(13.43 \%)$ had pre-existing depression. There were 64 (29.63\%) participants flagged for postpartum depression through the EPDS.

The bivariable and multivariable log-binomial regression models are presented in table 3. At the bivariable level, household income below the municipal median (RR: $3.66,95 \%$ CI:2.07 to 6.48 ) was significantly associated with perinatal IPV and EPDS score (RR: 1.04, 95\% CI: 1.00 to 1.10$)$ was marginally associated. In the multivariable models, household income below the municipal median (aRR: 3.24, 95\% CI: 1.87 to 5.59) was the strongest risk factor for any IPV and EPDS was marginally associated (aRR: $1.03,95 \%$ CI: 1.00 to 1.07 ). There was no independent association between maternal age (aRR: 0.99, 95\% CI: 0.94 to 1.04 ), nulliparity (aRR: $1.18,95 \%$ CI: 0.71 


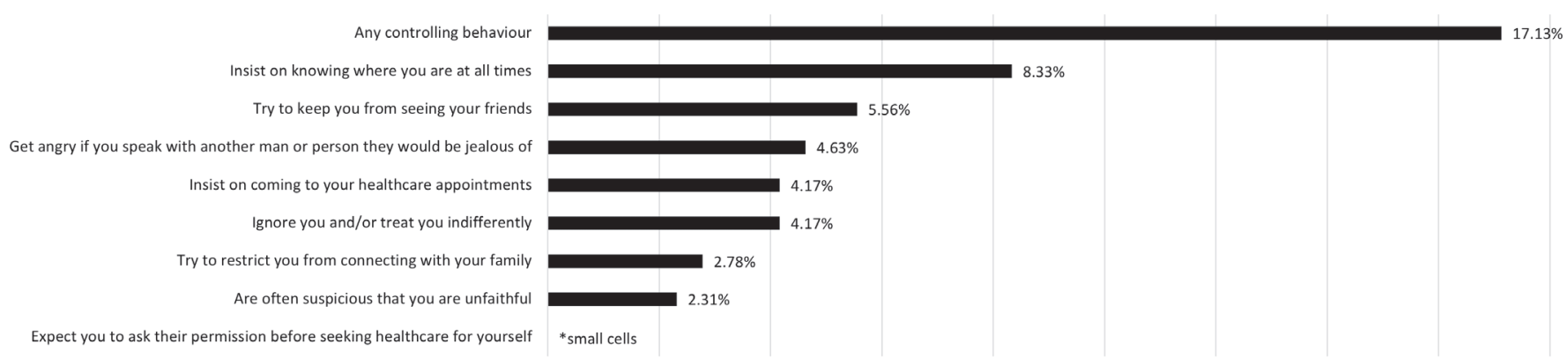

Figure 1 Different forms of regular controlling behaviour from partner during COVID-19 $(n=216)$. *measured using items from the WHO multicountry study on domestic violence.

to 1.97 ) or partner substance use increase (aRR: 0.73 , 95\% CI: 0.42 to 1.25 ) with IPV.

In sensitivity analyses, five individuals were identified who reported pre-pregnancy act-based forms of perinatal IPV alone; when removed from the sample, the estimates remained the same. The bivariable and multivariable results are available in online supplemental appendix 3.

\section{DISCUSSION}

In our study sample, almost a quarter $(24.07 \%)$ of the participants who gave birth during the COVID-19 pandemic reported some form of perinatal IPV, including regular controlling behaviours and act-based forms of IPV. Emotional abuse was the most common form of actbased perinatal IPV, however, cases of sexual and physical abuse were also identified. Household income was the strongest risk factor associated with perinatal IPV, and surprisingly, many hypothesised risk factors (eg, mental health, increased partner substance use, etc) were not significantly associated with perinatal IPV in this sample.
A strength of this study is the detailed breakdown of the different forms, timing and frequency of perinatal IPV, including revictimisation that happened in multiple perinatal time periods. ${ }^{47}$ The estimated prevalence of perinatal IPV in this study is higher than previous studies of the perinatal population (estimated to be an average of $3 \%-9 \%)$ and online surveys investigating violence during the COVID-19 pandemic (estimated between 10\% and 17\%). ${ }^{27} 48$ This may be influenced by the broad definition of perinatal IPV that we used (ie, two scales from the WHO multicountry study on domestic violence), that captures more forms of perinatal IPV compared with other scales. ${ }^{47-54}$ We chose an online survey as the method of administration, as online surveys have been shown to have higher rates of disclosure compared with face-to-face, paper or voice/telephone. ${ }^{49}$ Additionally, perinatal IPV included an observation window that included pre-pregnancy IPV that occurred before the COVID-19 pandemic began.

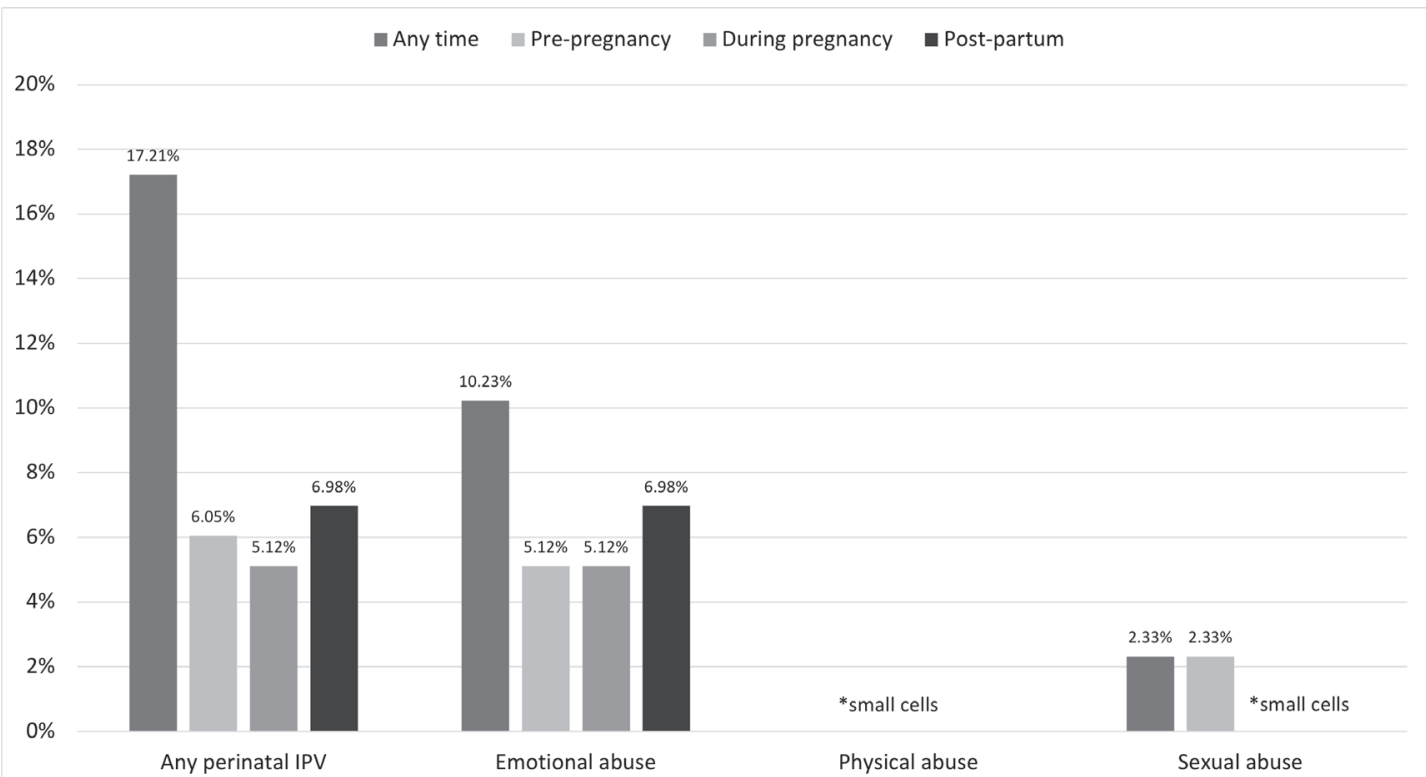

Figure 2 Forms and timing of act-based perinatal IPV ( $n=216)$. *participants can report multiple forms of IPV. Cases of physical and sexual abuse were identified, but suppressed because of small cell sizes. IPV, intimate partner violence. 
Table 2 General characteristics of study participants who have given birth since the COVID-19 pandemic began $(n=216)$

\begin{tabular}{|c|c|c|c|c|}
\hline \multirow[b]{2}{*}{ Variables } & \multicolumn{2}{|c|}{ Perinatal IPV } & \multirow{2}{*}{$\begin{array}{l}\text { Total } \\
\mathrm{N}=216\end{array}$} & \multirow[b]{2}{*}{$P$ value } \\
\hline & $\begin{array}{l}\text { Yes } \\
(n=52)\end{array}$ & $\begin{array}{l}\text { No } \\
(164)\end{array}$ & & \\
\hline \multicolumn{5}{|l|}{ Demographic characteristics } \\
\hline Maternal age in years (median, IQR) & $32(30-34)$ & $33(30-36)$ & $33(30-36)$ & 0.089 \\
\hline Infant age in days at time of interview (median, IQR) & $80(67-89)$ & $75(65-90)$ & $76(66-90)$ & 0.375 \\
\hline \multicolumn{5}{|l|}{ Month of delivery in 2020} \\
\hline March & $11(21.15)$ & $28(17.07)$ & $39(18.06)$ & 0.800 \\
\hline April & $21(40.38)$ & $61(37.20)$ & $82(37.96)$ & \\
\hline May & $13(25.00)$ & $51(31.10)$ & $64(29.63)$ & \\
\hline June & $7(13.46)$ & $24(14.63)$ & $31(14.35)$ & \\
\hline Nulliparous versus multiparous & $32(61.54)$ & $86(52.44)$ & $118(54.63)$ & 0.251 \\
\hline \multicolumn{5}{|l|}{ Race or ethnicity* } \\
\hline White people & $31(59.62)$ & $116(70.73)$ & $147(68.06)$ & 0.134 \\
\hline Asian-South/East/Southeast & $9(17.31)$ & $22(13.41)$ & $31(14.35)$ & 0.485 \\
\hline Middle Eastern & $8(15.38)$ & $10(6.10)$ & $18(8.33)$ & 0.035 \\
\hline Black people & $5(9.62)$ & $12(7.32)$ & $17(7.87)$ & 0.592 \\
\hline Another POC group & $9(17.31)$ & $16(9.76)$ & $25(11.57)$ & 0.138 \\
\hline Born in Canada versus immigrated & $34(65.38)$ & $125(76.22)$ & $159(73.61)$ & 0.122 \\
\hline $\begin{array}{l}\text { Marital status: married/common law versus single/another } \\
\text { status }\end{array}$ & $48(92.31)$ & $156(95.12)$ & $204(94.44)$ & 0.440 \\
\hline Completed college diploma or undergraduate degree & $44(84.62)$ & $144(87.80)$ & $188(87.04)$ & 0.551 \\
\hline \multicolumn{5}{|l|}{ Language(s) comfortable speaking* } \\
\hline Speaks English & $51(98.08)$ & $161(98.17)$ & $212(98.15)$ & 0.965 \\
\hline Speaks French & $19(36.54)$ & $55(33.54)$ & $74(34.26)$ & 0.691 \\
\hline Speaks another language & $9(17.31)$ & $20(12.20)$ & $29(13.43)$ & 0.346 \\
\hline \multicolumn{5}{|l|}{ Income } \\
\hline Dwelling owned versus rented & $29(55.77)$ & $122(74.39)$ & $151(69.91)$ & 0.011 \\
\hline Combined household income below Ottawa median $\dagger$ & $34(65.38)$ & $46(28.05)$ & $80(37.04)$ & $<0.001$ \\
\hline \multicolumn{5}{|l|}{ COVID-19-related household stress } \\
\hline Any household income loss & $19(36.54)$ & $45(27.44)$ & $64(29.63)$ & 0.211 \\
\hline Partner is essential worker & $12(23.08)$ & $43(26.22)$ & $55(25.46)$ & 0.650 \\
\hline Partner substance use increased & $12(23.08)$ & $33(20.12)$ & $45(20.83)$ & 0.648 \\
\hline \multicolumn{5}{|l|}{ Children's schedule } \\
\hline Children stopped going to school or daycare & $17(32.69)$ & $67(40.85)$ & $84(38.89)$ & 0.515 \\
\hline Children's schedule did not change & - & - & $14(6.48)$ & \\
\hline No children & $32(61.54)$ & $86(52.44)$ & $118(54.63)$ & \\
\hline \multicolumn{5}{|l|}{ Isolation due to COVID-19 restrictions } \\
\hline No baby shower or planned celebration & $29(55.77)$ & $70(42.68)$ & $99(45.83)$ & 0.099 \\
\hline Family member couldn't come to stay with me as planned & $30(57.69)$ & $78(47.56)$ & $108(50.00)$ & 0.203 \\
\hline Friends and family could not visit my new baby & $41(78.85)$ & $129(78.66)$ & $170(78.70)$ & 0.977 \\
\hline Missed out on community resources & $46(88.46)$ & $137(83.54)$ & $183(84.72)$ & 0.390 \\
\hline \multicolumn{5}{|l|}{ Mental health } \\
\hline Pre-existing anxiety & $13(25.00)$ & 30 (18.29) & $43(19.91)$ & 0.291 \\
\hline Pre-existing depression & $7(13.46)$ & $22(13.41)$ & $29(13.43)$ & 0.993 \\
\hline Postpartum depression (EPDS 13 cut-off) & $20(38.46)$ & $44(26.83)$ & $64(29.63)$ & 0.109 \\
\hline
\end{tabular}




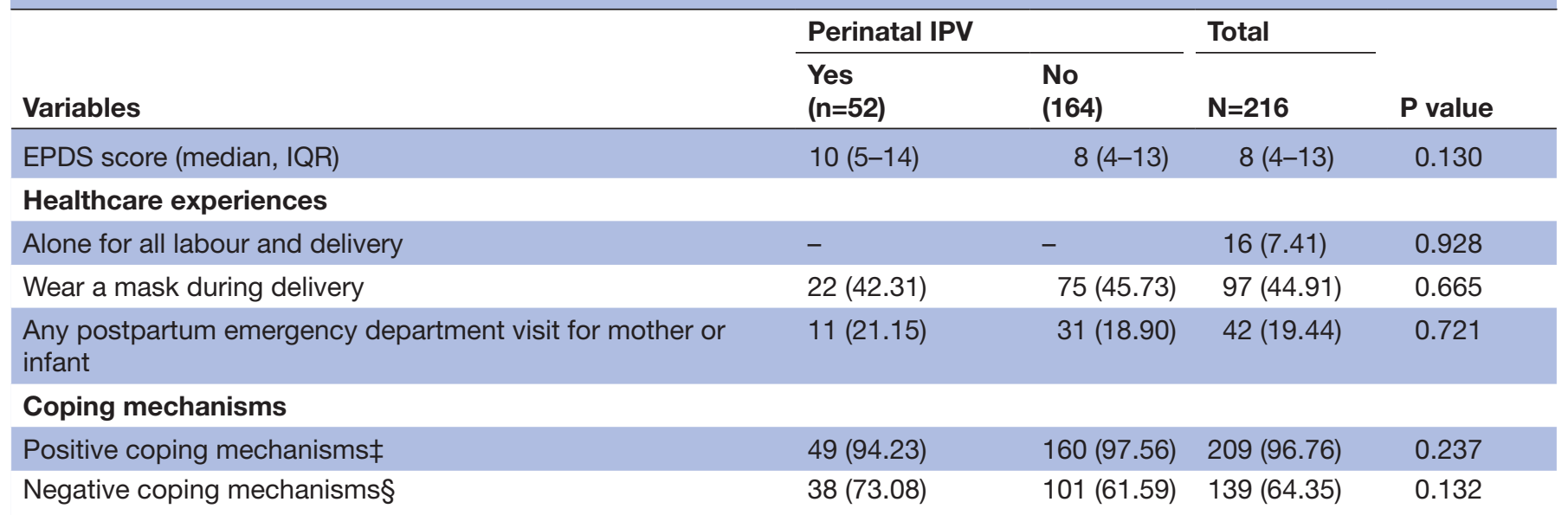

Cell sizes $\leq 5$ and are suppressed; $p$ values are calculated with $\mathrm{X}^{2}$ test for categorical variables and Wilcoxon rank-sum tests for continuous variables.

*Participants can pick multiple race/ethnic groups or languages.

†Missing data $=24$ cases.

$\ddagger$ Positive coping mechanisms include: yoga, exercising, donating to charity, connecting with friends/family.

$\S N e g a t i v e$ coping mechanisms include: sleeping more or less; over/undereating, acting aggressively.

EPDS, Edinburgh Postnatal Depression Survey; IPV, intimate partner violence; POC, person of colour.

In this analysis, the factor most strongly associated with perinatal IPV was socioeconomic status, contributing to over three times the risk of IPV. The association between income and violence is well established in the literature. ${ }^{55}$ Consistent with findings from national representative Canadian surveys,${ }^{56}$ almost $30 \%$ of participants reported household income loss because of COVID-19 protocols, and $25 \%$ reported that their partners were essential workers, but of interest, both of these factors were not significantly different by perinatal IPV status. This finding may be highlighting that the sustained stress of lower socioeconomic status poses a greater risk of perinatal IPV than the potential short-term effects of COVID-19-related changes in financial stability.

In the wake of COVID-19, attention has been drawn to the responsibility of healthcare practitioners for screening for and addressing IPV, including investigating circumstances around injuries. ${ }^{57}$ Within the obstetrical sphere, several commentaries have been written advocating for integrating IPV assessments into ambulatory gynaecological care, postpartum recovery, mental health and reproductive life planning. ${ }^{58-61}$ Systematic reviews and meta-analyses have identified that strongest sociodemographic factors associated with elevated risk of perinatal IPV include younger maternal age, single relationship status, visible minority status, lower socioeconomic status and substance use. ${ }^{562}$ Of interest within this study, several of the typical risk factors for IPV were not significantly different by perinatal IPV status. This lends support for the importance of universal IPV screening as risk factor-based screening may miss cases.

Despite the general pervasiveness of IPV and the increased risks posed by the COVID-19 pandemic, many healthcare providers are not well equipped to screen for violence or to take action if IPV is identified. ${ }^{6364}$ If children are in a home affected by violence, there are legal requirements to contact Children's Aid as part of due diligence. ${ }^{65}$ However, there are no such requirements for IPV, reinforcing a systemic barrier to violence prevention and care. During the pandemic

\begin{tabular}{|c|c|c|c|c|}
\hline Covariables & RR $(95 \% \mathrm{Cl})$ & $P$ value & aRR $(95 \% \mathrm{Cl})$ & $P$ value \\
\hline Maternal age in years (median, IQR) & 0.95 (0.90 to 1.00$)$ & 0.082 & 0.99 (0.94 to 1.04$)$ & 0.660 \\
\hline EPDS score (continuous) & $1.04(1.00$ to 1.10$)$ & 0.040 & $1.03(1.00$ to 1.07$)$ & 0.077 \\
\hline Nulliparous versus multiparous & 1.33 (0.76 to 2.32$)$ & 0.319 & $1.18(0.71$ to 1.97$)$ & 0.529 \\
\hline Partner substance use increased & 1.14 (0.60 to 2.17$)$ & 0.691 & $0.73(0.42$ to 1.25$)$ & 0.249 \\
\hline Household income below versus above Ottawa median* & 3.66 (2.07 to 6.48$)$ & $<0.001$ & 3.24 (1.87 to 5.59$)$ & $<0.001$ \\
\hline
\end{tabular}

*Missing data for household income were imputed by multiple imputation using chained equations and models were averaged across 10 imputed datasets.

†Outcome is defined as any reported regular controlling behaviour or act-based perinatal IPV (emotional, physical, sexual). aRR, adjusted RR; EPDS, Edinburgh Postnatal Depression Survey; IPV, intimate partner violence; RR, risk ratio. 
period, many services have introduced modifications to services, including closure of physical offices, which has limited access to interventional options. ${ }^{15}$ However, obstetrical departments are one of the few services that have continued to see patients in person. Pregnancy and the postpartum period is a window of opportunity to identify and support people experiencing violence who may have lost contact with other community services.

\section{Limitations}

We do not have information on those who did not consent to participate, which may introduce self-selection bias, non-response bias or residual confounding, all factors that may explain why several hypothesised risk factors were not significantly associated with perinatal IPV. All measures of IPV were self-reported and may underestimate the prevalence of IPV within this sample; however, the survey captured regular controlling behaviours that may not be perceived as abusive in addition to act-based forms of emotional abuse (eg, insulting, scaring, belittling, etc), physical abuse (eg, hitting, slapping, pushing), or sexual abuse (eg, forced sexual activity). While $94.4 \%$ were married/common law, we do not have information on the length of the relationship or if the participant had the same partner throughout the perinatal period. We did not have a comparison group of participants prior to COVID-19 and are unable to estimate the change in prevalence of perinatal IPV attributable to the increased stress of the COVID-19 pandemic. Additionally, this study was unable to evaluate associations between IPV and clinical outcomes as we did not have information on maternal or newborn outcomes.

\section{Conclusion}

Almost a quarter of this obstetrical study population reported some form of perinatal IPV, including IPV experienced pre-pregnancy, during pregnancy and post partum. Owing to COVID-19 lockdown measures, many participants reported household income loss, changes to childcare, and increased isolation during pregnancy and post partum; however, it was the strong social determinant of income that was most strongly associated with IPV. As the COVID-19 pandemic evolves, it is critical to prioritise the health and safety of the perinatal population in public health planning to ensure that households are fully supported and risks are mitigated.

\section{Author affiliations}

${ }^{1}$ Clinical Epidemiology Program, Ottawa Hospital Research Institute, Ottawa, Ontario, Canada

${ }^{2}$ Obstetrics and Gynecology, University of Ottawa, Ottawa, Ontario, Canada

${ }^{3}$ Faculty of Medicine, University of Ottawa, Ottawa, Ontario, Canada

${ }^{4}$ Mailman School of Public Health, Columbia University, New York City, New York,

USA

${ }^{5}$ School of Epidemiology and Public Health, University of Ottawa, Ottawa, Ontario,

Canada

${ }^{6}$ Children's Hospital of Eastern Ontario Research Institute, Ottawa, Ontario, Canada
${ }^{7}$ BORN Ontario, Children's Hospital of Eastern Ontario, Ottawa, Ontario, Canada ${ }^{8}$ Emergency Medicine, University of Ottawa, Ottawa, Ontario, Canada

\section{Twitter Katherine A Muldoon @K_Muldoon}

Acknowledgements We would like to acknowledge all the participants who took the time to respond to this survey. We thank Dr Jasmine Gandhi and Dr Mark Kaluzienski for support developing the perinatal mental health response study protocols.

Contributors KAM, KMD, RT, CB, OF, ALJH, RRW, KS and DE-C conceived and designed the study. KS, MCW and DE-C provided clinical expertise and content. RT conducted the statistical analyses; while KAM, DBF, YG, MSQM, DJC and SWW provided methodological and analytical expertise. $\mathrm{CB}, \mathrm{OF}$ and $\mathrm{KD}$ collected the data MAO'H-G is the patient partner. All authors critically reviewed the manuscript and approved the final version. KAM and DE-C have primary responsibility for the final content.

Funding This work was supported by The Ottawa Hospital Academic Medical Organization (TOHAMO): TOH-20-005.

Competing interests None declared.

Patient consent for publication Not required.

Ethics approval This study was approved by the Ottawa Health Sciences Network Research Ethics Board (protocol number: 20170390-01H). All participants provided informed verbal consent. All methods were performed in accordance with standard ethical guidelines and regulations (Declaration of Helsinki).

Provenance and peer review Not commissioned; externally peer reviewed.

Data availability statement Data are available upon reasonable request. The datasets generated and analysed during the current study are not publicly available due to the Ottawa Hospital privacy protocols; but with a data sharing agreement, de-identified data, the data dictionary and ethics protocol are available from the corresponding author.

Supplemental material This content has been supplied by the author(s). It has not been vetted by BMJ Publishing Group Limited (BMJ) and may not have been peer-reviewed. Any opinions or recommendations discussed are solely those of the author(s) and are not endorsed by BMJ. BMJ disclaims all liability and responsibility arising from any reliance placed on the content. Where the content includes any translated material, BMJ does not warrant the accuracy and reliability of the translations (including but not limited to local regulations, clinical guidelines, terminology, drug names and drug dosages), and is not responsible for any error and/or omissions arising from translation and adaptation or otherwise.

Open access This is an open access article distributed in accordance with the Creative Commons Attribution Non Commercial (CC BY-NC 4.0) license, which permits others to distribute, remix, adapt, build upon this work non-commercially, and license their derivative works on different terms, provided the original work is properly cited, appropriate credit is given, any changes made indicated, and the use is non-commercial. See: http://creativecommons.org/licenses/by-nc/4.0/.

\section{ORCID iDs}

Katherine A Muldoon http://orcid.org/0000-0002-0766-4294

Yanfang Guo http://orcid.org/0000-0003-4749-2033

Daniel J Corsi http://orcid.org/0000-0001-7063-3354

Shi Wu Wen http://orcid.org/0000-0002-7227-0283

Darine El-Chaar http://orcid.org/0000-0002-8266-0242

\section{REFERENCES}

1 Chandan JS, Taylor J, Bradbury-Jones C, et al. COVID-19: a public health approach to manage domestic violence is needed. Lancet Public Health 2020;5:30112:e309.

2 de Girolamo G, Cerveri G, Clerici M, et al. Mental health in the coronavirus disease 2019 Emergency-The Italian response. JAMA Psychiatry 2020;77:974-21.

3 Mazza M, Marano G, Lai C, et al. Danger in danger: interpersonal violence during COVID-19 quarantine. Psychiatry Res 2020;289:113046.

4 Roesch E. Violence against women during Covid-19 pandemic restrictions. Br Med J 2020.

5 Galea S, Merchant RM, Lurie N. The mental health consequences of COVID-19 and physical distancing: the need for prevention and early intervention. JAMA Intern Med 2020;180:E1-2. 
6 Breiding MJ, Basile KC, Smith SG. Intimate partner violence surveillance: uniform definitions and recommended data elements. Natl Cent Inj Prev Control Centers Dis Control Prev 2015;36:706-7.

7 UN Women. Policy Brief: The Impact of Covid-19 on Women, 2020. Available: https://www.unwomen.org/-/media/headquarters/ attachments/sections/library/publications/2020/policy-brief-theimpact-of-covid-19-on-women-en.pdf?la=en\&vs=5029

8 Usher K, Bhullar N, Durkin J. Family violence and COVID-19: increased vulnerability and reduced options for support. Int J Ment Health Nurs 2020

9 van Gelder N, Peterman A, Potts A, et al. COVID-19: reducing the risk of infection might increase the risk of intimate partner violence. EClinicalMedicine 2020;21:100348.

10 Violence Against Women Learning Network. COVID-19 \& GenderBased Violence in Canada : Key Issues and Recommendations. London, Ontario, 2020.

11 World Health Organization. Global status report on violence prevention, 2014

12 Hahn CK, Gilmore AK, Aguayo RO, et al. Perinatal intimate partner violence. Obstet Gynecol Clin North Am 2018;45:535-47.

13 Solomon MD, McNulty EJ, Rana JS. The Covid-19 pandemic and the incidence of acute myocardial infarction. N Engl J Med 2020:10-12.

14 Frankfurter C, Buchan TA, Kobulnik J, et al. Reduced rate of hospital presentations for heart failure during the COVID-19 pandemic in Toronto, Canada. Can J Cardiol 2020;36:1680-4.

15 Muldoon KA, Denize KM, Talarico R. COVID-19 pandemic and violence : rising risks and decreasing urgent care-seeking for sexual assault and domestic violence survivors. BMC Med 2021:1-9.

16 Olding J, Zisman S, Olding C, et al. Penetrating trauma during a global pandemic: changing patterns in interpersonal violence, self-harm and domestic violence in the Covid-19 outbreak. Surgeon 2021;19:e9-13.

17 Morris D, Rogers M, Kissmer N. Impact of lockdown measures implemented during the COVID-19 pandemic on the burden of trauma presentation to a regional emergency department in KwaZulu natal, South Africa. African J Emerg Med 2020. doi:10.1016/j. afjem.2020.06.005

18 Garstang J, Debelle G, Anand I, et al. Effect of COVID-19 lockdown on child protection medical assessments: a retrospective observational study in Birmingham, UK. BMJ Open 2020;10:1-6. doi:10.1136/bmjopen-2020-042867

19 Barbara G, Facchin F, Micci L, et al. COVID-19, Lockdown, and intimate partner violence: some data from an Italian service and suggestions for future approaches. $J$ Womens Health 2020;29:1239-42.

20 Piquero AR, Riddell JR, Bishopp SA. Staying safe? A short-term analysis of COVID-19 on Dallas domestic violence. Am J Crim Justice 2020. doi:10.1007/s12103-020-09531-7

21 Leslie E, Wilson R. Sheltering in place and domestic violence: evidence from calls for service during COVID-19. J Public Econ 2020;189:104241.

22 Sutherland M, McKenney M, Elkbuli A. Gun violence during COVID-19 pandemic: paradoxical trends in New York City, Chicago, Los Angeles and Baltimore. Am J Emerg Med 2021;39:225-6.

23 Statistics Canada. Police-Reported crime incidents down during the early months of the pandemic, while domestic disturbance calls increase. Ottawa, 2020.

24 Payne JL, Morgan A, Piquero AR. COVID-19 and social distancing measures in Queensland, Australia, are associated with short-term decreases in recorded violent crime. J Exp Criminol 2020:1-25.

25 Hodgkinson T, Andresen MA. Show me a man or a woman alone and I'll show you a Saint: changes in the frequency of criminal incidents during the COVID-19 pandemic. J Crim Justice 2020;69:101706.

26 Mohler G, Bertozzi AL, Carter J, et al. Impact of social distancing during COVID-19 pandemic on crime in Los Angeles and Indianapolis. J Crim Justice 2020;68:101692.

27 Lindberg L, VandeVusse D, Mueller A. Early Impacts of the COVID-19 Pandemic: Findings from the 2020 Guttmacher Survey of Reproductive Health Experiences. New York, 2020. www.guttmacher. org

28 Jetelina KK, Knell G, Molsberry RJ. Changes in intimate partner violence during the early stages of the COVID-19 pandemic in the USA. Inj Prev 2021;27:1-5.

29 SE I, Jenkins M, Gendall P. Psychological distress, anxiety, family violence, suicidality, and wellbeing in New Zealand during the COVID-19 lockdown : A cross-sectional study. PLoS One 2020;15:1-19.

30 Abou Ghayda R, Li H, Lee KH, et al. COVID-19 and adverse pregnancy outcome: a systematic review of 104 cases. J Clin Med 2020;9:3441.
31 Di Mascio D, Khalil A, Saccone G, et al. Outcome of coronavirus spectrum infections (SARS, MERS, COVID-19) during pregnancy: a systematic review and meta-analysis. Am J Obstet Gynecol MFM 2020;2:100107.

32 Novoa RH, Quintana W, Llancarí P. Maternal clinical characteristics and perinatal outcomes among pregnant women with coronavirus disease 2019. A systematic review. Travel Med Infect Dis 2020;2021:101919.

33 Vardhelli V, Pandita A, Pillai A, et al. Perinatal COVID-19: review of current evidence and practical approach towards prevention and management. Eur J Pediatr 2021;180:1009-31.

34 Zaigham M, Andersson O. Maternal and perinatal outcomes with COVID-19: a systematic review of 108 pregnancies. Acta Obstet Gynecol Scand 2020;99:823-9.

35 Yee J, Kim W, Han JM. Clinical manifestations and perinatal outcomes of pregnant women with COVID-19: a systematic review and meta-analysis. Sci Rep 2020;10:1-7.

36 Di Toro F, Gjoka M, Di Lorenzo G, et al. Impact of COVID-19 on maternal and neonatal outcomes: a systematic review and metaanalysis. Clin Microbiol Infect 2021;27:36-46.

37 Zambrano LD, Ellington S, Strid P, et al. Update: Characteristics of Symptomatic Women of Reproductive Age with LaboratoryConfirmed SARS-CoV-2 Infection by Pregnancy Status - United States, January 22-October 3, 2020. MMWR Morb Mortal Wkly Rep 2020;69:1641-7.

38 Hessami K, Romanelli C, Chiurazzi M. COVID-19 pandemic and maternal mental health: a systematic review and meta-analysis. $J$ Matern Neonatal Med 2020.

39 Preis H, Mahaffey B, Heiselman C, et al. Pandemic-related pregnancy stress and anxiety among women pregnant during the coronavirus disease 2019 pandemic. Am J Obstet Gynecol MFM 2020;2:100155

40 Government of Canada. Coronavirus disease 2019 (COVID-19): epidemiology update, 2020. Available: https://health-infobase. canada.ca/covid-19/epidemiological-summary-covid-19-cases.html [Accessed June 3rd, 2020].

41 The Ottawa Hospital, The Ottawa Hospital. High-Risk twin pregnancy during COVID-19 pandemic, 2020. Available: https://ohfoundation. $\mathrm{ca} /$ be-inspired/high-risk-twin-pregnancy-during-covid-19-pandemic/ [Accessed June 3rd, 2020].

42 Garcia-Moreno C, Jansen H, Ellsberg M. WHO Multi-Country Study on Women's Health and Domestic Violence Against Women: Report on the First Results. World Heal Organ 2005:55-89.

43 Ottawa Public Health. Socio-Demographic data and population projects. General population health, 2020. Available: https:// www.ottawapublichealth.ca/en/reports-research-and-statistics/ sociodemographics.aspx [Accessed June 3rd, 2020].

44 Statistics Canada. Distribution of total income by census family type and age of older partner, parent, or individual, 2017. Available: https://www150.statcan.gc.ca/n1/en/catalogue/1110001201 [Accessed 18 Dec 2020].

45 SAS Institute Inc. Sas Institute Inc, 2017.

46 Azur MJ, Stuart EA, Frangakis C, et al. Multiple imputation by chained equations: what is it and how does it work? Int J Methods Psychiatr Res 2011;20:40-9.

47 Rodrigues T, Rocha L, Barros H. Physical abuse during pregnancy and preterm delivery. Am J Obstet Gynecol 2008;198:171.e1-171.e6.

48 Hui DSC, Chan PKS. Severe acute respiratory syndrome and coronavirus. Infect Dis Clin North Am 2010;24:619-38.

49 Fraga S, Lucas R, Barros $\mathrm{H}$. A comparison of methods to assess intimate partner abuse 1 year postpartum. Ann Epidemiol 2014;24:404-6.

50 MacMillan HL, Wathen CN, Jamieson E, et al. Approaches to screening for intimate partner violence in health care settings: a randomized trial. JAMA 2006;296:530-6.

51 Taillieu TL, Brownridge DA, Tyler KA, et al. Pregnancy and intimate partner violence in Canada: a comparison of victims who were and were not abused during pregnancy. J Fam Violence 2016;31:567-79.

52 Daoud N, Urquia ML, O'Campo P, O'campo P, et al. Prevalence of abuse and violence before, during, and after pregnancy in a national sample of Canadian women. Am J Public Health 2012;102:1893-901.

53 Graham-Kevan N, Archer J. Investigating Three Explanations of Women's Relationship Aggression. Psychol Women Q 2005;29:270-7.

54 McFarlane J, Parker B, Soeken K, et al. Assessing for abuse during pregnancy. severity and frequency of injuries and associated entry into prenatal care. JAMA 1992;267:3176-8

55 Pastor-Moreno G, Ruiz-Pérez I, Henares-Montiel J, et al. Intimate partner violence during pregnancy and risk of fetal and neonatal death: a meta-analysis with socioeconomic context indicators. Am J Obstet Gynecol 2020;222:123-33. 
56 Statistics Canada. Canadian Perspectives Survey Series 1 : Impacts of COVID-19, 2020. Available: https://www150.statcan.gc.ca/n1/en/ daily-quotidien/200408/dq200408c-eng.pdf?st=AhCEAktc

57 Bradley N, DiPasquale AM, Dillabough K. Health care practitioners' responsibility to address intimate partner violence related to the COVID-19 pandemic. Can Med Assoc J 2020:1-2.

58 Cohen MA, Powell AM, Coleman JS, et al. Special ambulatory gynecologic considerations in the era of coronavirus disease 2019 (COVID-19) and implications for future practice. Am J Obstet Gynecol 2020;223:372-8.

59 Sadler M, Leiva G, Olza I. COVID-19 as a risk factor for obstetric violence. Sex Reprod Health Matters 2020;28:1785379.

60 Choi K, Records K, Low LK. Promotion of Maternal-Infant mental health and Trauma-Informed care during the coronavirus disease 2019 pandemic. J Obstet Gynecol Neonatal Nurs 2020:1-7.
61 Tang K, Gaoshan J, Ahonsi B. Sexual and reproductive health $(\mathrm{SrH})$ : a key issue in the emergency response to the coronavirus disease (COVID- 19) outbreak. Reprod Health 2020;17.

62 Costa D, Hatzidimitriadou E, loannidi-Kapolou E, et al. Male and female physical intimate partner violence and socio-economic position: a cross-sectional international multicentre study in Europe. Public Health 2016;139:44-52.

63 Rabin RF, Jennings JM, Campbell JC, et al. Intimate partner violence screening tools: a systematic review. Am J Prev Med 2009;36:439-45.

64 Doherty OL, Hegarty K, Ramsay J. Screening women for intimate partner violence in healthcare settings (review). Cochrane Database Syst Rev 2015;2015. doi:10.1002/14651858.CD007007.pub3

65 Government of Ontario. Child, youth and family services act, 2017, chapter 14. Canada, 2017: 1-166. 